\title{
Encephalitis lethargica : la COVID-19 de longue durée du siècle dernier?
}

\author{
Citation : CMAJ 2021 September 20;193:E1468-70. doi : 10.1503/cmaj.210807-f
}

Voir la version anglaise de l'article ici : www.cmaj.ca/lookup/doi/10.1503/cmaj.210807

es cliniciens ont commencé à décrire des maladies chroniques postvirales, comme l'« hystérie postgrippale ", dès les années $1890^{1}$. Mais leurs traités étaient souvent vagues et truffés de généralisations douteuses. C'est grâce au développement massif des systèmes de santé publics, à l'époque de la pandémie de grippe de 1918, qu'on a enfin commencé à reconnaître les séquelles chroniques des viroses. Dans ses observations sur des cas aigus d'Encephalitis lethargica en 1917, Constantin von Economo (neuroanatomiste autrichien) présentait les symptômes suivants : fièvre, céphalées, paralysie oculaire et somnolence exceptionnelle. Beaucoup ont associé le syndrome au virus responsable de la pandémie de grippe de 1918 , mais le lien de causalité n'a jamais été ni confirmé ni infirmé ${ }^{2}$. Cette incertitude a motivé une intensification des travaux sur les formes chroniques de l'encéphalite, même après l'essoufflement de la pandémie dans les années $1920^{3}$.

En Amérique, les chercheurs ont organisé des conférences de consensus et des projets de recherche bibliographique colossaux, pour finalement publier en 1929 un rapport qui compilait les résultats de plus de 4000 publications sur quelque 50000 cas aigus d'encéphalite dans le monde ${ }^{4}$. Ils ont aussi mis au point un processus cinématographique de ralenti extrême pour étudier les tremblements parkinsoniens chez les personnes atteintes d'encéphalite chronique ${ }^{5}$. En France, des experts ont critiqué les autorités de la santé publique, dont les activités de surveillance n'ont pas su repérer les dizaines de milliers de cas aigus qu'ils soupçonnaient dans la population

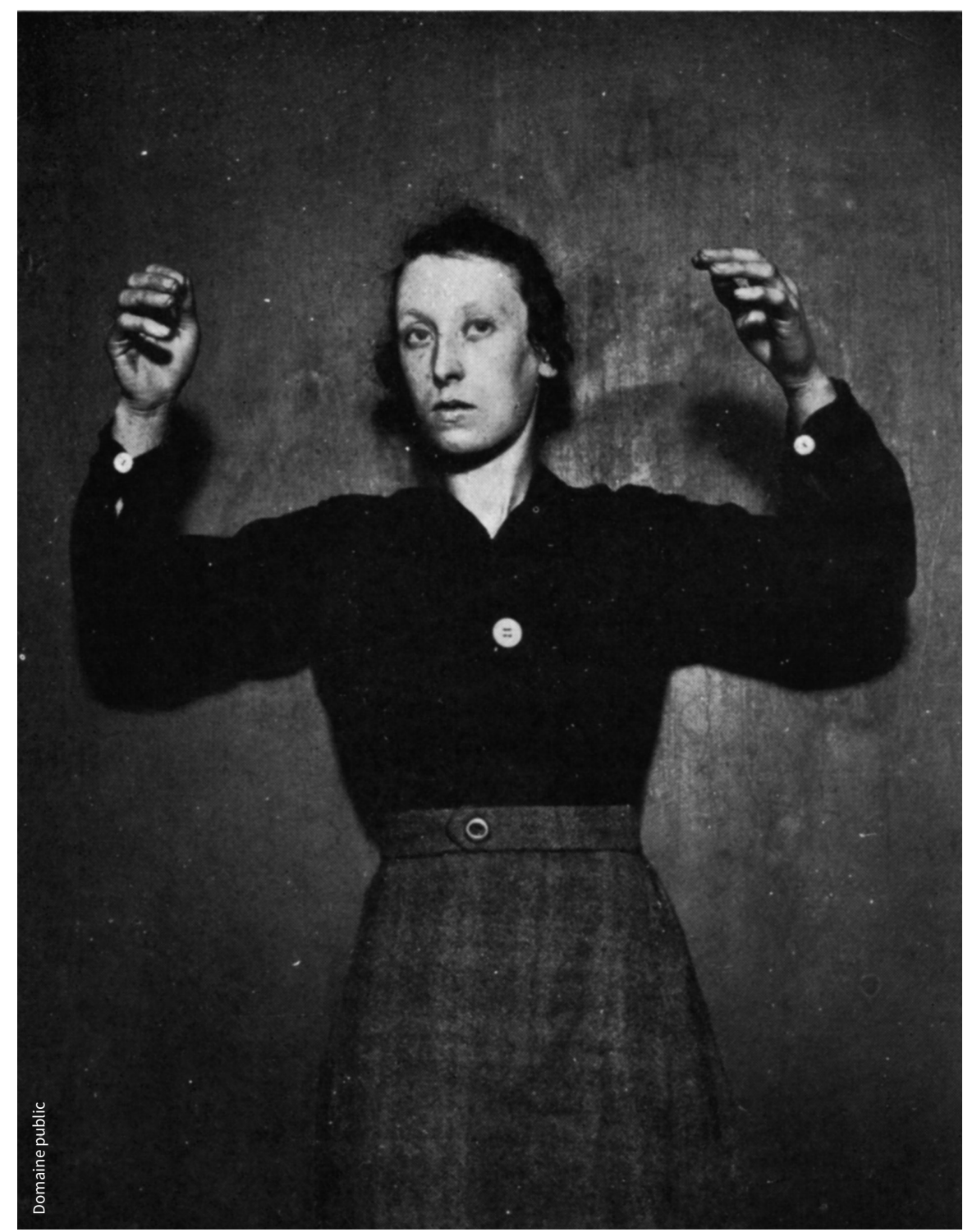

Figure 1 : Photographie (planche XV) tirée d'Epidemic encephalitis (1924) d'A.J. Hall. Ce type d'images venait renforcer la démarche initiale du clinicien face à l'état post-encéphalite de ses patients, qu'il caractérisait par une perte de tonalité affective (visage inexpressif) et de volonté (Hall a noté que, sans qu'on le lui demande, la patiente ne résistait pas au repositionnement de ses bras et qu'elle ne faisait aucun effort pour les ramener à la position initiale une fois la photo prise). 
générale ${ }^{6}$. En Allemagne, une poignée de neuropsychiatres ont réalisé des entrevues approfondies chez leurs patients atteints dans l'espoir de mieux comprendre certains troubles connexes, comme la schizophrénie catatonique $\mathrm{e}^{7}$.

Le système de santé public de pointe de la Grande-Bretagne a permis la détection d'un nombre élevé d'" encéphalites épidémiques ", ce qui a suscité des préoccupations répandues dans la société quant aux effets chroniques. Le choix d'une nouvelle dénomination découlait de la crainte grandissante que le terme "encéphalite léthargique » constitue à la fois un ensemble de symptômes mal défini et une véritable épidémie. Malgré une surveillance étroite de la santé publique, les autorités britanniques répétaient souvent qu'il était probable que la plupart des cas ne soient pas rapportés et que les séquelles à long terme de l'encéphalite (fatigue chronique, insomnie, tremblements, troubles de la démarche, troubles oculaires, changements radicaux de comportements contribuant à la délinquance juvénile, etc.) puissent accompagner les cas aigus légers.

Originaire de Sheffield, un pôle industriel d'environ un demi-million de personnes, Arthur J. Hall est devenu la référence britannique en ce qui a trait à la maladie et à son importance. Hall (18661951) jouissait d'une réputation nationale d'éducateur brillant et d'administrateur avisé ayant modernisé à lui seul la faculté de médecine de sa ville natale. Il s'est intéressé aux grappes de cas d'encéphalite qui ont émergé à Sheffield au début de 1924, et sa clinique spécialisée de l'hôpital Sheffield Royal a accueilli des centaines de patients atteints en quelques mois seulement. À la fin de l'année, il avait amassé suffisamment de rapports d'observations pour produire une monographie abondamment illustrée qui a fait école. Complété par une affiche détachable, son ouvrage détaillait les symptômes variés et complexes de l'encéphalite pour aider les omnipraticiens du pays à détecter et à signaler les cas de cette maladie qui, selon Hall et tant d'autres, était très répandue, mais passait sous le radar de la santé publique ${ }^{8}$.

L'assiduité avec laquelle il a suivi la trajectoire de ses patients après la publication d'Epidemic encephalitis en 1924 témoigne de l'émergence de pratiques d'observation clinique empathiques parallèlement aux changements aux politiques et aux services médicaux britanniques. Les effets de l'expansion de l'assurance maladie des travailleurs et l'accès à un soutien psychologique plus complet pour les écoliers et les parents ont été ressentis dans toute la société britannique dans les années 1920 . Les historiens ont montré comment ces améliorations ont transformé les soins de santé primaires pour accorder une plus grande place à la psyché et aux émotions du patient ${ }^{9,10}$. L'implication de Hall auprès de ses patients a évolué de façon semblable. Ainsi, en 1924, la voix de ces derniers était totalement exclue de sa monographie. Les photographies de patients à différents stades de paralysie catatonique mettaient en exergue leur impuissance et leur volonté affaiblie (figure 1). Par la suite, Hall a accordé une attention croissante à ses patients dans les années 1920 et 1930, malgré le recul des éclosions d'encéphalite. Conservés aux archives de l'Université de Sheffield, les dossiers du médecin illustrent ses efforts pour consigner tous les changements vécus par ses patients postencéphalitiques, dans la mesure où il les observait à leur insu peiner par exemple à maîtriser leurs tremblements assez bien pour allumer une cigarette après une promenade en vélo ou à manger une soupe sans aide au club médical de Sheffield fondé par Hall des années auparavant.

Les tremblements et la rigidité n'étaient pas les seuls symptômes post-encéphalite ayant piqué la curiosité de Hall. Ses notes cliniques indiquent qu'il a fidèlement suivi le parcours de ses patients pendant des décennies à l'aide d'articles de journaux, de chroniques nécrologiques et d'entrevues auprès des familles pour tenter de voir si les séquelles de la maladie avaient joué un rôle - et dans quelle mesure - dans leur décès accidentel ou par suicide. Même si Hall en est venu à constater que ces 2 causes de décès étaient d'une fréquence improbable, sa démarche reposait moins sur la statistique que sur un désir sincère de catégoriser avec exactitude les incapacités postencéphalite pour mieux soigner les patients à long terme.

Plus Hall étudiait l'état post-encéphalite, plus il valorisait les témoignages des patients. Ses archives en contiennent plusieurs, méticuleusement documentés, qui montrent sa détermination à continuer de recueillir ces données. La teinture de belladone était souvent utilisée pour traiter l'état post-encéphalite en présence de symptômes parkinsoniens et semblait paradoxalement plus efficace que d'autres extraits plus puissants, dont l'atropine ou la scopolamine. Hall expliquait ce phénomène en se basant directement sur les témoignages des patients. Ceux-ci ont décrit une détente euphorique qui leur permettait de se mouvoir sans y apporter autant d'attention et d'effort, ce qui les incitait à bouger davantage et accentuait ainsi les effets psychologiques d'abord limités de la belladone. Hall se fiait aussi aux dires de ses patients sur leur état psychologique et affectif pour trancher sur l'administration d'atropine : si la fonction psychologique était gravement compromise, il concluait que le recours à l'atropine n'était pas indiqué.

L'encéphalite a souvent été décrite comme une affection des plus mystérieuses qui se manifestait sans raison apparente et qui, lentement et insidieusement, privait ses victimes de tout espoir d'avenir. L'attention accrue qu'a portée Hall aux propos de ses patients sur leur situation difficile peut faire penser à une réponse pleine d'empathie à une grande tragédie humaine. Les recherches historiques nous aident toutefois à mieux comprendre la démarche dans le contexte historique et géographique. Grâce à sa réputation locale et nationale, Hall a créé un système à l'écoute des patients qui faisait résonner leur voix. Il ne voyait pas vraiment de distinction entre rendre les nouveaux systèmes britanniques empathiques et les rendre efficients. Plus que jamais auparavant, les systèmes ont été conçus pour prendre soin des Britanniques tout au long de leur vie. II va sans dire que les assurances privées existaient depuis longtemps déjà et que Hall était motivé à poursuivre l'observation de ses patients post-encéphalitiques en raison de son implication non seulement dans les institutions publiques, mais aussi dans le recrutement de clients pour les assurances privées - une pratique courante chez les médecins britanniques de l'époque ${ }^{11}$ qui l'a poussé à considérer la progression des patients à long terme. 
La curiosité du médecin a profité directement aux patients atteints d'encéphalite, quoi qu'on pense de ses intérêts variés, qui dépassaient la simple empathie pour impliquer le réseau complexe de forces scientifiques, culturelles et politiques présentes en Grande-Bretagne pendant l'entre-deux-guerres. On ferait peut-être bien de s'en inspirer pour attiser la curiosité des professionnels qui suivent et qui soignent les patients atteints de la COVID-19 de longue durée alors que nous repensons les systèmes de santé pour surmonter les défis à long terme grâce à des gains de sensibilité, d'adaptabilité et d'efficacité.

\section{Kenton Kroker PhD}

Programme en santé et société, Département des sciences sociales, Faculté des arts libéraux et des études professionnelles, Université York, Toronto, Ont.

\section{Références}

1. Honigsbaum M, Krishnan L. Taking pandemic sequelae seriously: from the Russian influenza to COVID-19 long-haulers. Lancet 2020;396:1389-91.
2. Vilensky JA, editor. Encephalitis lethargica: during and after the epidemic. New York: Oxford University Press; 2011.

3. Kroker K. Configuring epidemic encephalitis as a national and international neurological concern: technique, technology, therapy. In: The history of the brain and mind sciences. Casper ST, Gavrus D, editors. Rochester (NY): University of Rochester Press; 2017:77-106.

4. Epidemic encephalitis : etiology, epidemiology, treatment. Report of a survey by the Matheson Commission. New York: Columbia University Press; 1929.

5. Kroker K. Creatures of reason? Picturing viruses at the Pasteur Institute during the 1920s. In: Crafting immunity: working histories of clinical immunology. Kroker K, Keelan J, Mazumdar PMH, editors. Aldershot (UK): Ashgate Publishers; 2008:145-63.

6. Bernard L, Renault J. L'enquête épidémiologique du ministre de l'Hygiène sur l'encéphalite léthargique en France. Bull Acad Méd (Paris) III Ser 1920;83:470-4.

7. Foley PJ. The Encephalitis lethargica patient as a window on the soul. In: The neurological patient in history. Jacyna LS, Casper ST, editors. Rochester (NY): University of Rochester Press; 2012:184-211.

8. Hall AJ. Epidemic encephalitis (Encephalitis lethargica). Bristol (UK): John Wright \& Sons; 1924.
9. Thomson M. Psychological subjects: identity, culture, and health in twentieth-century Britain. Oxford (UK): Oxford University Press; 2006.

10. Hayward R. The transformation of the psyche in British primary care, 1870-1970. London (UK): Bloomsbury; 2014.

11. Dupree MW. Other than healing: medical practitioners and the business of life assurance during the nineteenth and early twentieth centuries. Soc Hist Med 1997;10:79-103.

Cet article a été commandé et a été révisé par des pairs.

Intérêts concurrents : Kenton Kroker a reçu une subvention de recherche du Conseil de recherches en sciences humaines du Canada. Aucun autre intérêt concurrent n'a été déclaré.

Propriété intellectuelle du contenu : Il s'agit d'un article en libre accès distribué conformément aux modalités de la licence Creative Commons Attribution (CC BY-NC-ND 4.0), qui permet l'utilisation, la diffusion et la reproduction dans tout médium à la condition que la publication originale soit adéquatement citée, que l'utilisation se fasse à des fins non commerciales (c.-à-d., recherche ou éducation) et qu'aucune modification ni adaptation n'y soit apportée. Voir : https://creativecommons.org/licenses/by -nc-nd/4.0/deed.fr. 